Pacific Journal of Mathematic 


\title{
ON COVERING SPACES AND GALOIS EXTENSIONS
}

\section{N. ChILDS}

\begin{abstract}
Let $X$ be a connected compact Hausdorff space, and $G$ a finite abelian group. In this note we obtain a short exact sequence (Theorem 1) which describes the group of isomorphism classes of regular covering spaces of $X$ with group $G$. The sequence is derived as an immediate translation of a similar sequence involving the group of commutative Galois extensions with group $G$ of $C(X)$, the ring of complex-valued continuous functions on $X$.
\end{abstract}

The translation is obtained in part by showing (Theorem 2) that there is an equivalence between the category of finite covering spaces of $X$ and the dual of the category of separable $C(X)$-algebras which are finitely generated projective $C(X)$-modules. This equivalence may be known to students of [8], but I am unaware of any reference for it, so we sketch a proof here.

1. The sequence. Let $X, C(X)$ be as above, and let $G$ be a finite group. A not necessarily connected covering space $Y$ over $X$ is called regular with group $G$ if $G$ acts as a fixed point free group of homeomorphisms of $Y$ which preserve the covering map. Denote by $\operatorname{Cov}(X, G)$ the set of isomorphism classes of regular covering spaces of $X$ with group $G$ (where $Y, Z$, two covering spaces with group $G$, are isomorphic if there is a homeomorphism from $Y$ to $Z$ which commutes with the covering maps and the action of $G)$. Denote by Pic $(X)$ the group (under tensor product of fibers) of isomorphism classes of line bundles on $X$. Denote by $H_{s}^{2}(G, U(C(X)))$ the subgroup of $H^{2}(G, U(C(X))$ ) (group cohomology, with $G$ acting trivally on $U(C(X))$ ) which is the image of the symmetric 2-cocycles-those cocycles $f$ from $G \times G$ into the units of $C(X)$ which satisfy $f(s, t)=f(t, s)$ for all $s, t$ in $G$.

Theorem 1. Let $G$ be a finite abelian group. Then $\operatorname{Cov}(X, G)$ has an abelian group structure so that the following sequence of abelian groups is exact:

$$
0 \rightarrow H_{s}^{2}(G, U(C(X))) \rightarrow \operatorname{Cov}(X, G) \rightarrow \operatorname{Hom}(G, \operatorname{Pic}(X)) \rightarrow 0 .
$$

Proof. If $G$ is a group of order $n$ and $R$ is a commutative ring with unity, a commutative $R$-algebra $S$ is a Galois extension of $R$ with group $G$ if $G$ acts as a group of $R$-algebra automorphisms of $S, R$ is the fixed ring under the action of $G$, and $([3,1.3 \mathrm{f}])$ for each 
maximal ideal $m$ of $S$ and $\sigma \neq 1$ in $G$, there is an $s$ in $S$ so that $\sigma(s)-s \notin m$. Two Galois extensions $S^{\prime}, S^{\prime \prime}$ with group $G$ are isomorphic if there is an $R$-algebra isomorphism $S^{\prime} \rightarrow S^{\prime \prime}$ which preserves the action of $G$. If $G$ is an abelian group, Harrison [9] has shown that the set of isomorphism classes of commutative Galois extensions with group $G$ forms an abelian group, $\operatorname{Comm}(R, G)$. Viewing a Galois extension of $R$ with group $G$ as a rank one projective $R[G]$-module defines a homomorphism from $\operatorname{Comm}(R, G)$ to $\operatorname{Pic}(R[G])$ (the group under tensor product of isomorphism classes of rank one projective $R[G]$-modules), whose kernel consists of the set $N B(R, G)$ of isomorphism classes of commutative Galois extensions with normal basis. If $R$ has no idempotents but 0 and 1 and contains $1 / n$ and a primitive $n$th root of unity, then the image is isomorphic to $\operatorname{Hom}(G, \operatorname{Pic}(R))$ [6, Theorem 9], so that we have the short exact sequence

$\left(^{*}\right) \quad 0 \rightarrow N B(R, G) \rightarrow \operatorname{Comm}(R, G) \rightarrow \operatorname{Hom}(G, \operatorname{Pic}(R)) \rightarrow 0$.

We set $R=C(X)$ in $\left(^{*}\right)$ and translate. Pic $(C(X)) \cong \operatorname{Pic}(X)$ by Swan [11]; $\left.N B(C(X), G) \cong H_{s}^{2}(G, U(X))\right)$ by Theorems 2.2 and 4.4 of [5]. It suffices to show that $\operatorname{Comm}(C(X), G) \cong \operatorname{Cov}(X, G)$. This will be a corollary of Theorem 2 .

2. The equivalence. $X, C(X)$ are as above.

THEOREM 2. There are category equivalences between the category of finite covering spaces of $X$, the dual of the category of separable $C(X)$-algebras which are finitely generated projective $C(X)$-modules, and the category of nonramified affine coverings of $\operatorname{Spec}(C(X))$ [8]. The first equivalence is induced by: if $Y$ is a covering space, $Y \rightarrow C(Y)$; if $S$ is a separable $R$-algebra, $S \rightarrow \operatorname{Max}(S)$. The functor from the first to the third sends $S$ to $\operatorname{Spec}(S)$.

Here $\operatorname{Max}(S)$ is the space of maximal ideals of $S$ with the Stone topology (= the topology induced on the geometric points from the Zariski topology on Spec $(S))$.

Corollary. $\operatorname{Cov}(X, G)$ is an abelian group isomorphic to $\operatorname{Comm}(C(X), G)$.

Proof of corollary. If $R=C(X), S=C(Y)$, it follows easily from the definition of Galois extension given above that $S$ is a Galois extension of $R$ with group $G$ if and only if $Y$ is a regular covering space of $X$ with group $G$. Hence there is a bijection between $\operatorname{Cov}(X, G)$ and $\operatorname{Comm}(R, G)$. The group structure on $\operatorname{Cov}(X, G)$ is 
the one induced from $\operatorname{Comm}(R, G)$.

Concerning Theorem 2, we have included the category of nonramified affine coverings of $\operatorname{Spec}(C(X))$ only to make more explicit the relationship with [8]. We shall show only that the correspondences $Y \rightarrow C(Y), S \rightarrow \operatorname{Max}(S)$ give inverse bijections between the objects of the first two categories; the proof of the rest of the theorem is straightforward and will be omitted.

In what follows, the phrase " $S$ is a finitely generated projective $R$-algebra" will mean that $S$ is an $R$-algebra which is finitely generated and projective as an $R$-module.

Proof of Theorem 2. We recall some facts about a compact Hausdorff space $X$ (see [7]): The topology of $X$ has a basis consisting of the complements of zero sets of continuous functions on $X$ (" cozero sets"). [7, 3.2, p. 38]. If $f$ is a continuous function let $Z(f)=$ the zeros of $f$ and $V(f)=X-Z(f)$. For any closed set $F$, if $\left.C(X)\right|_{F}$ denotes the restriction to $F$ of the continuous functions on $X$, then $\left.C(X)\right|_{F}=C(F) \quad[7,3.11(\mathrm{c})$, p. 43]. For any open set $V=V(f)$, $\left.C(X)\right|_{V}=C(X)_{f}$, the localization of $C(X)$ with respect to the multiplicative set consisting of the powers of $f$. $\operatorname{Max}(C(X))$, the set of maximal ideals of $C(X)$, is in one-to-one correspondence with the points of $X$, since any maximal ideal of $C(X)$ is of the form $\{f$ in $C(X) \mid f(p)=0\}$ for some point $p$ of $X$. If $\operatorname{Max}(C(X))$ is given the Stone topology: basic closed sets are of the form $\{x \mid f$ is in $x\}=Z(f)$ for $f$ in $C(X)$, then $\operatorname{Max}(C(X))$ is homeomorphic to $X[7,4.9, \mathrm{p} .58]$.

Assume now that $X$ is a compact Hausdorff space and $Y$ a finite covering space, that is, there is a continuous map $p: Y \rightarrow X$ and for each $x$ in $X$ a neighborhood $U$ of $x$ (a canonical neighborhood) such that $p^{-1}(U)$ is the disjoint union of a finite number $n$ of open sets of $Y$ each homeomorphic to $U$.

Fix an $x$ in $X$, let $U$ be a canonical neighborhood, and let $F$ be a closed subneighborhood. Then $p^{-1}(F)$ is a disjoint union of closed sets of $Y$ each homeomorphic to $F$, so $C\left(p^{-1}(F)\right) \cong C(F)^{n}$ (the product as rings of $n$ copies of $C(F)$ ). Let $V=V(f)$ be a cozero set containing $x$ and contained in $F$. Then $V_{Y}(f)=V_{Y}(f \circ p)=p^{-1}\left(V_{X}(f)\right) \subseteq p^{-1}(F)$ is a disjoint union of open sets of $Y$ each homeomorphic to $V_{X}(f)$. So $C(Y)_{f}=\left.C(Y)\right|_{V_{Y}(f)}=\left.C\left(p^{-1}(F)\right)\right|_{p\left(V_{X}(f)\right)} ^{-1}$ is the ring of continuous functions on a finite disjoint union of open sets each homeomorphic to $V_{X}(f)$, whence $C(Y)_{f} \cong\left(C(X)_{f}\right)^{n}$. Thus for each maximal ideal $x$ of $C(X)$ there is a $f$ not in $x$ so that $C(Y)_{f}$ is a finitely generated projective separable $C(X)_{f}$-algebra. By $[4, \S 5]$ and $[1] C(Y)$ is therefore a finitely generated projective separable $C(X)$-algebra.

For the other direction, we need a 
LEMMA. Let $S$ be a finitely generated projective separable $R$ algebra, $R=C(X)$. Then for each $x$ in $X$ there is a neighborhood $V(h)$ of $x$ so that $S_{h} \cong\left(R_{h}\right)^{n}$, a product as rings of $n$ copies of $R_{h}$.

Proof of lemma. $R_{x}$, the localization of $R$ with respect to the maximal ideal $x$, is a local ring, so by Theorems 3.1,2.2 and 2.8 of [10], $S_{x}=R_{x}[\theta]$, where $\theta$ satisfies a polynomial $f(t)=t^{n}+a_{1} t^{n-1}+\cdots+a_{n}$ with coefficients in $R_{x}$ and with $n$ distinct roots $s_{1}, \ldots, s_{n}$ in $R / x \cong \boldsymbol{C}$. Let $\theta=a / b$ with $a$ in $S, b$ in $R-x$, let $k$ be the product of the denominators of the coefficients of $f(t)$, and let $d$ be the discriminant [10] of $f(t)$. Then on $V(g)$ with $g=b d k, R_{g}[\theta]$ is a separable $R_{g^{-}}$ algebra contained in $S_{g}$, and is a finitely generated projective $R_{g^{-}}$ module of the same rank as $S_{g}$. So $S_{g}$ is a projective $R_{g}[\theta]$-module of rank one. But since $S_{g}$ is a finitely generated projective $R_{g}[\theta]-$ algebra, $R_{g}[\theta]$ is a $R_{g}[\theta]$-direct summand of $S_{g}$. Thus $S_{g}=R_{g}[\theta]$. Since $f(t)$ has coefficients in $R_{g}, S_{g} \cong R_{g}[t] /(f(t))$.

Claim: There exists a subneighborhood $V=V(h)$ of $V(g)$ containing $x$, and continuous functions $r_{1}, \ldots, r_{n}$ in $C(X)$ so that on $V$, $f(t)=\prod_{i=1}^{n}\left(t-r_{i}\right)$. This follows from the implicit function theorem applied to the function $F(\bar{a}, t)=t^{n}+a_{1} t^{n-1}+\cdots+a_{n}$ at $\bar{a}=\bar{a}(x)=$ $\left(a_{1}(x), \ldots, a_{n}(x)\right) \in C^{n}, t=s_{i}$. For since $F(\bar{a}(x), t)$ has $n$ distinct roots, the partial derivative $F_{t}\left(\bar{a}(x), s_{i}\right) \neq 0$, so there exists a neighborhood $U$ of $\bar{a}(x)$ in $C^{n}$ and continuous functions $t_{i}: U \rightarrow C$ such that $F\left(\bar{a}, t_{i}(a)\right)=0$ for all $\bar{a}$ in $U$. Since $t_{i}(\bar{a}(x))=s_{i} \neq s_{j}=t_{j}(\bar{a}(x))$ for all $i \neq j$ we can pick $U$ so small that $t_{i}(U) \cap t_{j}(U)=\varnothing$ for all $i \neq j$. If we set $\chi$ : $V(g) \rightarrow C^{n}$ by $\chi(y)=\bar{a}(y)$, then $\chi$ is a continuous function, so $V(g) \cap$ $\chi^{-1}(U)$ is a neighborhood of $x$ on which there exist continuous functions $\widetilde{r}_{i}=t_{i} \circ \chi, i=1, \ldots, n$, with disjoint images, which are roots of $f$. Hence there is a basic open subneighborhood $V(h)$ (containing $x$ ) of a closed subreighborhood of $V(g) \cap \chi^{-1}(U)$ and $n$ elements $r_{1}, \ldots, r_{n}$ of $C(X)$ so that on $V(h), f(t)=\prod_{i=1}^{n}\left(t-r_{i}\right)$. The lemma follows easily.

Suppose now that $S$ is a finitely generated projective separable $R$-algebra, and set $Y=\operatorname{Max}(S)$. Put the Stone topology on $Y$. Let $p: Y \rightarrow X$ by $p(y)=y \cap R$, a maximal ideal since $S$ is integral over $R$ ([2]).

Let $x$ be a point of $X$. By the lemma there exists an $h$ in $C(X)$ so that $S_{h}=\left(R_{h}\right)^{n}$, a product as rings of copies of $R_{h}$. Then $V(h)$ is easily seen to be a canonical neighborhood of $x$, and $p$ is continuous, so that $Y$ is a covering space of $X$.

It is easy to verify that the topology defined on $Y$ makes $Y$ into a compact Hausdorff space.

If $Y$ is a covering space and a compact Hausdorff space, then $Y=\operatorname{Max}(C(Y))$ as topological spaces by $[7,3.6, \mathrm{p} .40]$. On the other 
hand, given $S$, a finitely generated projective separable $R$-algebra, $S$ is clearly contained in $C(\operatorname{Max}(S))$. Replacing $\operatorname{Max}(S)$ by each of its connected components as necessary, we may assume that $S$ and $C(\operatorname{Max}(S))$ have no nontrivial idempotents. Embed $C(\operatorname{Max}(S))$ in a finitely generated projective Galois extension $T$ of $R$ containing no idempotents but 0 and 1 (possible by [10,1.13]). By the fundamental theorem of Galois theory ([3, Theorem 2.3]) $S$ is the fixed ring of some subgroup of $\operatorname{Aut}_{R}(T)$. But any group which fixes $S$ fixes $C(\operatorname{Max}(S))$, whence $S=C(\operatorname{Max}(S))$. This completes the proof of Theorem 2.

\section{REFERENCES}

1. Bourbaki, Algebra Commutative, Chapitre II.

2. - Chapitre III.

3. S. Chase, D. Harrison, A. Rosenberg, Galois theory and Galois cohomology of commutative rings, Memoirs Amer. Math. Soc., 52 (1965), 15-33.

4. S. Chase, A. Rosenberg, Amitsur cohomology and the Brauer Group, Memoirs Amer. Math. Soc., 52 (1965), 34-79.

5. - A theorem of Harrison, Kummer theory, and Galois algebras, Nagoya J. Math., 27 (1966), 663-685.

6. L. Childs, Abelian Galois extensions of rings containing roots of unity, Illinois J. Math., (to appear).

7. L. Gillman, M. Jerison, Rings of Continuous Functions, Princeton, Van Nostrand, 1960.

8. A. Grothendieck, Geometric formelle et geometrie algebrique, Sem. Bourbaki 182 (1959).

9. D. Harrison, Abelian extensions of commutative rings, Memoirs Amer. Math. Soc., 52 (1965), 1-14.

10. G. Janusz, Separable algebras over commutative rings, Trans. Amer. Math. Soc., 122 (1966), 461-479.

11. R. G. Swan, Vector bundles and projective modules, Trans. Amer. Math. Soc., 105 (1962), 267-277.

Received February 19, 1970.

State University of New York, Albany 



\section{PACIFIC JOURNAL OF MATHEMATICS}

\section{EDITORS}

H. SAMELSON

Stanford University

Stanford, California 94305

C. R. Новву

University of Washington

Seattle, Washington 98105
J. DUGundJI

Department of Mathematics

University of Southern California

Los Angeles, California 90007

RICHARD ARENS

University of California

Los Angeles, California 90024

\section{ASSOCIATE EDITORS}
E. F. BECKENBACH
B. H. NeumanN
F. WOLF
K. YOSHIDA

\section{SUPPORTING INSTITUTIONS}
UNIVERSITY OF BRITISH COLUMBIA
CALIFORNIA INSTITUTE OF TECHNOLOGY
UNIVERSITY OF CALIFORNIA
MONTANA STATE UNIVERSITY
UNIVERSITY OF NEVADA
NEW MEXICO STATE UNIVERSITY
OREGON STATE UNIVERSITY
UNIVERSITY OF OREGON
OSAKA UNIVERSITY
UNIVERSITY OF SOUTHERN CALIFORNIA
STANFORD UNIVERSITY
UNIVERSITY OF TOKYO
UNIVERSITY OF UTAH
WASHINGTON STATE UNIVERSITY
UNIVERSITY OF WASHINGTON
AMERICAN MATHEMATICAL SOCIETY CHEVRON RESEARCH CORPORATION TRW SYSTEMS
NAVAL WEAPONS CENTER 


\section{Pacific Journal of Mathematics}

\section{Vol. 37, No. $1 \quad$ January, 1971}

Gregory Frank Bachelis and Haskell Paul Rosenthal, On unconditionally

converging series and biorthogonal systems in a Banach space .........

Richard William Beals, On spectral theory and scattering for elliptic

operators with singular potentials .........................

J. Lennart (John) Berggren, Solvable and supersolvable groups in which every element is conjugate to its inverse ........................ 21

Lindsay Nathan Childs, On covering spaces and Galois extensions ..........

William Jay Davis, David William Dean and Ivan Singer, Multipliers and

unconditional convergence of biorthogonal expansions..............

Leroy John Derr, Triangular matrices with the isoclinal property ............

Paul Erdős, Robert James McEliece and Herbert Taylor, Ramsey bounds for

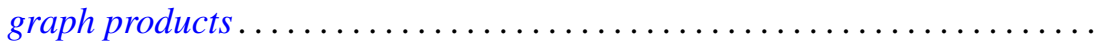

Edward Graham Evans, Jr., On epimorphisms to finitely generated

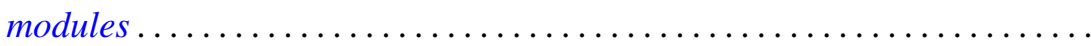

Hector O. Fattorini, The abstract Goursat problem ................. 51

Robert Dutton Fray and David Paul Roselle, Weighted lattice paths .........

Thomas L. Goulding and Augusto H. Ortiz, Structure of semiprime $(p, q)$

radicals ...........................................

E. W. Johnson and J. P. Lediaev, Structure of Noether lattices with join-principal maximal elements ....

David Samuel Kinderlehrer, The regularity of minimal surfaces defined over

slit domains

Alistair H. Lachlan, The transcendental rank of a theory. .

Frank David Lesley, Differentiability of minimal surfaces at the boundary ...

Wolfgang Liebert, Characterization of the endomorphism rings of divisible torsion modules and reduced complete torsion-free modules over complete discrete valuation rings....

Lawrence Carlton Moore, Strictly increasing Riesz norms.

Raymond Moos Redheffer, An inequality for the Hilbert transform ...

James Ted Rogers Jr., Mapping solenoids onto strongly self-entwined,

circle-like continua..........................

Sherman K. Stein, B-sets and planar maps ................... 217

Darrell R. Turnidge, Torsion theories and rings of quotients of Morita

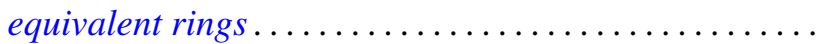

Fred Ustina, The Hausdorff means of double Fourier series and the principle of localization ................................

Stanley Joseph Wertheimer, Quasi-compactness and decompositions for arbitrary relations.

Howard Henry Wicke and John Mays Worrell Jr., On the open continuous images of paracompact $\check{C}$ ech complete spaces... 